\title{
Keeping the Patent Internal Mammary Artery out of Harm's way at Future Reoperation
}

\author{
Mahmoud Abdelaziz* and Jitendra Parmar \\ University Hospital Coventry and Warwickshire, UK
}

*Corresponding author: Mahmoud M Abdelaziz, University Hospitals Coventry and Wawickshire NHS Trust Cardiothoracic Department Coventry, UK.

Received Date: October 04, 2018

Published Date: October 15, 2018

\begin{abstract}
Keeping a patent left internal mammary artery (LIMA) at re-operation is a challenging task. This crucial graft can be damaged at sternal re-entry for redo surgery in various steps. We devised a technique to minimise this risk. The result of this technique is a patent LIMA routed through a trans pleural tunnel, away from the midline and protected by a layer of the parietal pleura, pleuropericardial fat and pericardium.
\end{abstract}

\section{Introduction}

With increase in long term survival after coronary artery bypass surgery and Left Internal Mammary Artery (LIMA) patency reaching up to $96 \%$ at 15 years, re-sternotomy for repeat cardiac surgery in the presence of a patent LIMA is not unusual [1]. Today, decades after the introduction of Coronary Artery Bypass Grafting (CABG) as a routine procedure in many medical centres, 1 in every 5 to 7 patients who have undergone one CABG may need a reoperation [2]. Ivert et al concluded that of 29 patents internal mammary artery (IMA) grafts in their cohort, 11 (38\%) were damaged at reoperation with a noticeable raise in perioperative myocardial infarction (40\%) and mortality [3-4].

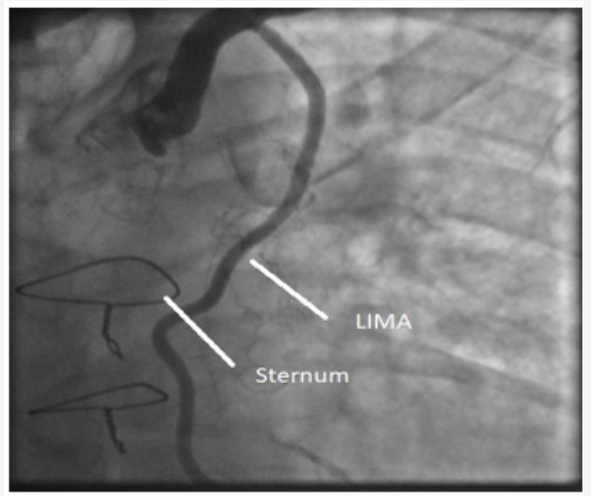

Figure 1: Coronary arteriogram during left internal mammary artery injection. Note the immediate medial substernal course of the unprotected harvested left internal thoracic artery, making it vulnerable to injury during subsequent redo-sternotomy.
Avoiding damage to a patent LIMA is a challenge to the surgical team as the final position of the graft is variable. It often lies behind the sternum when displaced medially, due to lung reexpansion at the end of the operation (Figure 1). Damage to such a crucial graft can happen at any stage of the reoperation such as at resternotomy using the oscillating saw, or when dissecting the left sided retrosternal adhesions, or when manipulating the heart and mobilising the LIMA pedicle.

\section{Technique}

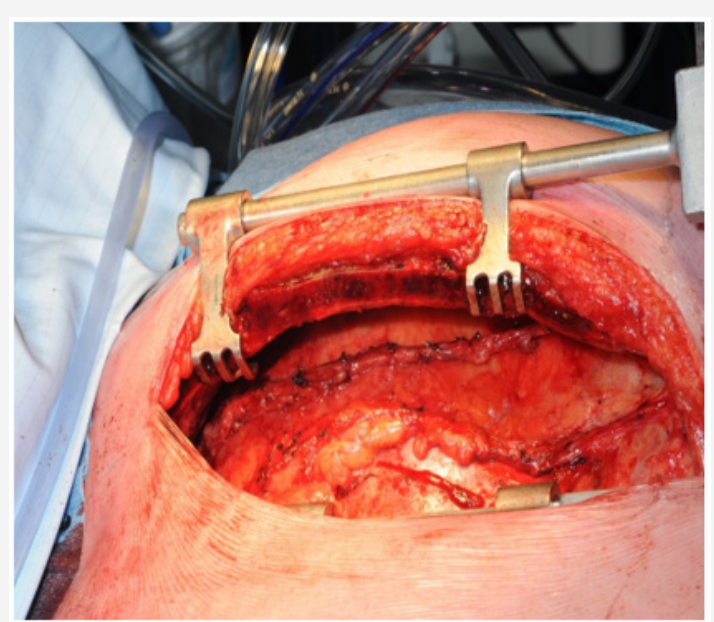

Figure 2: Extra pleural harvest of the LIMA to the level of the left subclavian artery. 
Median sternotomy incision is performed as routine at the primary operation; the mediastinal fat lateral to the left lobe of the thymus is cleared up to the pleuro-pericardial reflection laterally. Extra pleural harvest of the LIMA is performed to the level of the left subclavian artery (Figure 2)

The LIMA is divided distally after heparinisation and tested for patency. A trans pleural tunnel is created through the parietal pleura by creating two openings. A proximal opening at the parietal pleural edge near the apex of the left upper lobe and another opening near the origin of the LIMA at the pleura-pericardial reflection (Figures 3, 4).

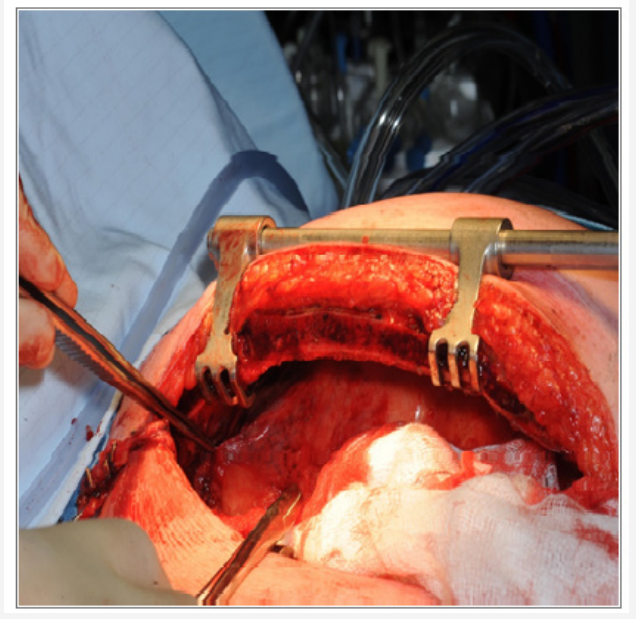

Figure 3: Creating a proximal opening at the parietal pleural edge near the apex of the left upper lobe.

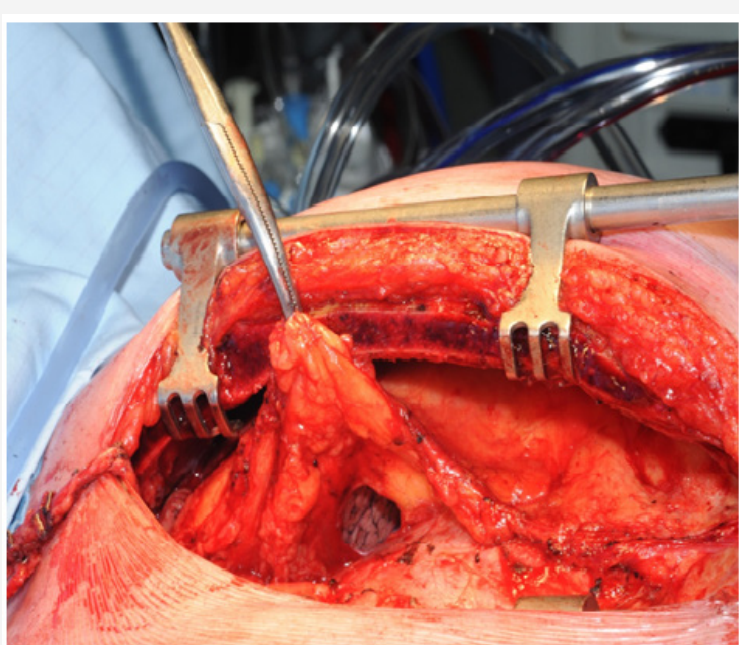

Figure 4: Creating a distal opening at the pleura-pericardial reflection.

The LIMA is routed through the pleural cavity from the apical pleural opening to the pleuro-pericardial opening using a Semba clamp. A vertical incision in the pericardium will bring the LIMA into the mediastinum and is usually optimally placed for left anterior descending coronary artery or the diagonal or the obtuse marginal coronary anastomosis (Figure 5) With gentle re-inflation of the lungs the end results of this manoeuvre are a LIMA graft lying within the pleural cavity medial to the upper lobe of the left lung and protected by a layer of pleura (Figure 6).

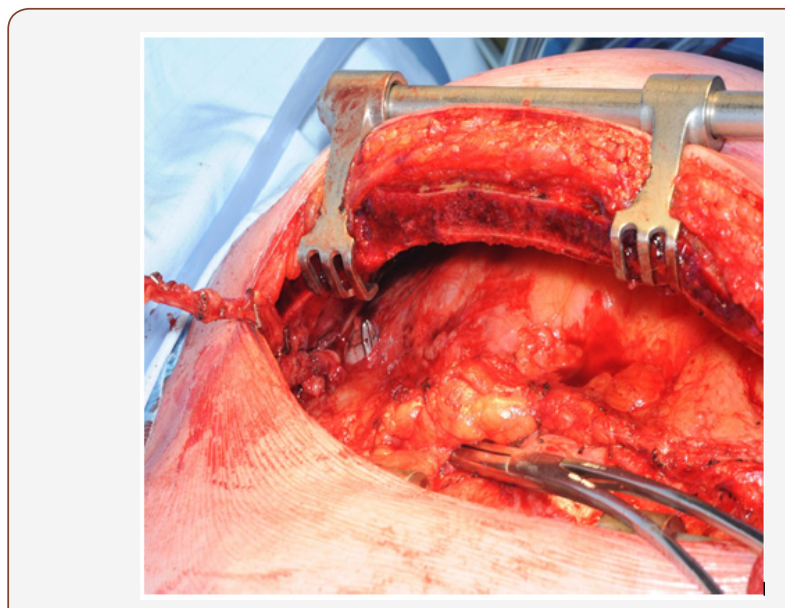

Figure 5: The LIMA is routed through the pleural tunnel created.

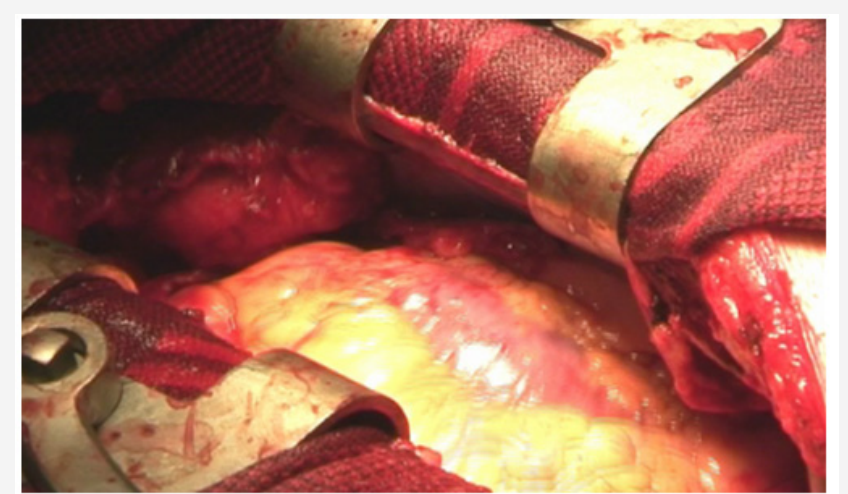

Figure 6: Final position of the LIMA graft.

Hence, we have displaced the LIMA from the midline, we are more accurate of its position in re-sternotomy and it is also protected from adherence to the sternum. Risk of damage to the LIMA at re-sternotomy and mediastinal dissection is therefore minimal. At re-operation, the left sided retrosternal tissue can be dissected without fear of injuring the LIMA. The patent LIMA can easily be accessed by incising the pleura.

\section{Comment}

In this position, the patent LIMA is not at risk of injury during sternal re-entry and mediastinal dissection for redo CABG procedures. This technique is simple, adds only a few minutes to the operative procedure, uses readily available native tissues in their anatomic location, and avoids heterologous biological membranes (such as bovine pericardium) or foreign bodies (such as polytetrafluoroethylene [5]) and their added cost and inherent risk of inflammatory foreign body reaction, calcification, and infection.

\section{References}

1. Tatoulis J, Buxton BF, Fuller JA (2004) Patencies of 2127 arterial to coronary conduits over 15 years. Ann Thorac Surg 77(1): 93-101.

2. Shapira I, Isakov A, Heller I, Topilsky M, Pines A (1999) Long-term follow-up after coronary artery bypass grafting reoperation. Chest 115(6): 1593-1597.

3. Ivert TS, Ekeström S, Péterffy A, Welti R (1988) Coronary artery reoperations. early and late results in 101 patients. Scand J Thorac Cardiovasc Surg 22(2): 111-118. 
4. Gillinov AM, Casselman FP, Lytle BW, Blackstone EH, Parsons EM, et al. (1999) Injury to patent left internal thoracic artery graft at coronary reoperation. Ann Thorac Surg 67(2): 382-386.
5. Zehr KJ, Lee PC, Poston RS, Gillinov AM,Hruban RH, etal.(1993) Protection of the internal mammary artery pedicle with polytetrafluoroethylene membrane. J Cardiac Surg 8(6): 650-655. 